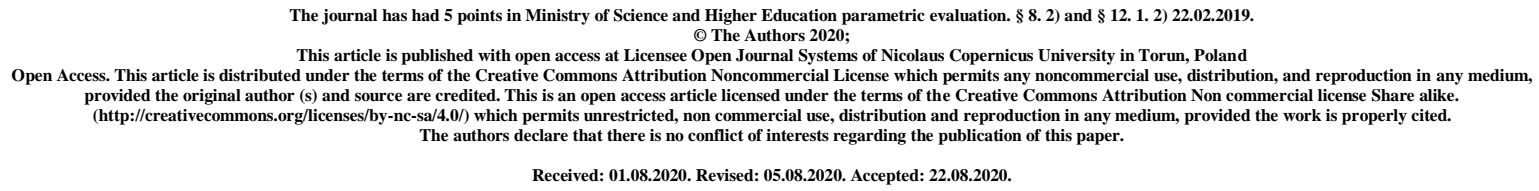

\title{
E-cigarettes, is this alternative safe?
}

\section{Małgorzata Romaniuk², Konrad Suswal', Mateusz Komisarczuk², Weronika Tuszyńska ${ }^{2}$, Karol Krupa ${ }^{2}$, Wojciech Zygmunt ${ }^{1}$, Janusz Milanowski ${ }^{1}$}

1. Chair and Department of Pneumology, Oncology and Allergology, Medical University of Lublin.

2. Pulmonology Student Research Group at the Clinic of Pneumology, Oncology and Allergology, Medical University of Lublin.

Authors:

1. M. Romaniuk, goszarom@gmail.com, ORCID: 0000-0001-8838-6291

2. K. Suswał, konrad.suswal92@gmail.com, ORCID: 0000-0001-5226-8779

3. M. Komisarczuk, mateusz.komisarczuk@gmail.com

4. W. Tuszyńska,weron.stud28@gmail.com

5. K. Krupa,k.krupa95@gmail.com

6.W.Zygmunt, wojciech.zygmunt92@gmail.com

7. J. Milanowski, janusz.milanowski@umlub.pl 


\begin{abstract}
Electronic cigarettes are often presented as a safer alternative to classic cigarettes. We present an overview of the latest data on the safety of using e-cigarettes and their impact on the respiratory and cardiovascular systems. The statement that e-cigarettes are much less harmful seems too hasty due to the relatively short presence of e-cigarettes on the market. We need more years of research on large groups of patients to help us answer the question whether ecigarettes are a better and safer form of smoking.
\end{abstract}

Key words: classic cigarettes; e-cigarettes; lung cancer.

\title{
Introduction
}

Smoking has been known to mankind for thousands of years. Initially, it played a role in religious and magical ceremonies [1]. In the era of great geographical discoveries, tobacco was one of the first things brought by the Spaniards from the New World. In the mid-18th century, a cigarette rolling machine was constructed, which was a breakthrough in the availability of cigarettes and led to the rapid popularization of this addiction [2].

According to the World Health Organization (WHO), about 1.3 billion people currently smoke tobacco. WHO records about 8 million deaths from smoking each year, of which 7 million are the result of the addiction to smoking, and over a million are the result of passive smoking [3]. It is estimated that tobacco is responsible for $80 \%$ of lung cancer cases [4]. Smoking increases the likelihood of cancer of the mouth, throat, larynx, esophagus, kidney, bladder, pancreas and cervix. Inhaling the smoke from smoking by another person (so-called secondhand smoke) also contributes to the development of lung cancer in adult nonsmokers [5].

The most popular form of tobacco smoking are classic cigarettes, other forms are less popular, such as hookah, cigars, cigarillos, rolls, beedi, kretek, and nowadays - new tobacco and nicotine products. [3] The willingness to expand the market and gain new customers has prompted tobacco manufacturers to seek alternative forms of smoking. One of them is electronic cigarettes (e-cigarettes), invented in 2003 by the Chinese pharmacist Hon Lik [6].

The e-cigarette consists of a battery, a cartridge containing an inhalation solution (a so-called liquid) and an atomizer in which the liquid is heated and turns into an aerosol inhaled by the smoker. The inhalation solution usually contains propylene glycol, nicotine, glycerin and flavoring substances [7]. Electronic cigarettes are often presented as a safer option compared to classic cigarettes, but we do not have more detailed research into the effects of their longterm use. We present an overview of the reports constituting an introduction to the discussion on this topic.

\section{Effect of e-cigarette fluid on health}

So far, studies have been conducted in the United States to evaluate the potential of ecigarettes as a source of toxic metals. It has been proven that the liquid that is used in ecigarettes contains elements such as: cadmium $(\mathrm{Cd})$, chromium $(\mathrm{Cr})$, lead $(\mathrm{Pb})$, manganese (Mn) and nickel (Ni). 
Such studies were conducted by the authors Hess, Olmedo et al. [8] Who searched for the most toxic and potentially carcinogenic metals that would help assess the harmfulness of ecigarettes.

However, significant variability in the concentrations of nickel and chromium, manganese and lead was found between producers. Cadmium concentrations were relatively low [8].

Diacetyl, 2,3-pentanedione and acetoin are other compounds found in exhaled smoke when an e-cigarette is burned. They are used in the production of many other foods and for the production of a wide variety of flavors (e.g. caramel, Scotch whiskey, pina colada, strawberry). In the work of Allen et al. [9] 51 types of flavors of e-cigarette cartridges sold by leading cigarette companies have been assessed. The aim of the study was to estimate the total mass of diacetyl, pentanedione and acetoin that are released during combustion in air. At least one of these aromatizing chemicals was detected in 47 of the 51 aromas tested. Diacetyl alone was detected in 39 of 51 flavors, while diacetyl with 2,3-pentanedione was detected in 21 flavors simultaneously. There was a strong association between loss of functionality and the development of lung diseases such as obliterative bronchiolitis, and exposure to the buttery flavoring, most of which is diacetyl. Interestingly, two other aromatics, acetoin and 2,3pentanedione, were also present in considerable amounts as combustion products.

The influence of electronic cigarettes on the respiratory system

The FDA (Food and Drug Administration) recognizes the flavoring compounds (e.g. diacetyl) contained in e-cigarette liquids as safe for oral consumption, but does not take into account the effects of these substances entering the body through inhalation. According to Clapp et al., flavoring compounds have toxic properties and irritate the respiratory tract, disrupting their physiological defense mechanisms [10].

Reinikovaite et al. [11] conducted a study to check whether a long-term exposure (5 weeks) to e-cigarette aerosol has the same detrimental effect on the structure and vascular system of the lungs as tobacco smoke on the example of an in vivo rat model. Based on plasma nicotine concentrations, rats exposure to e-cigarette aerosol and classical tobacco smoke was comparable to human exposure to e-cigarettes and conventional cigarettes. Exposure of rats to both electronic cigarette aerosol and cigarette smoke in the test chambers for 5 weeks led to significant $(\mathrm{p}<0.01)$ lung remodeling as a result of emphysema compared to the control group. Despite the fact that the concentrations of nicotine in the blood and cotinine (a breakdown product of nicotine) in the urine were higher in the case of classic cigarettes, the destruction of the lungs in both groups was similar. It was shown that lung damage was similar in both cases, although e-cigarettes are poorer in terms of composition, and classic cigarettes contain about 7,000 different chemical components] [11].

Studies in adolescents have shown an increased risk of developing respiratory symptoms. A survey of approximately 45,000 teenagers in Hong Kong found that e-cigarette use in the past 30 days was associated with increased reporting of chronic cough or sputum production (odds ratio $2.1,95 \%$ confidence interval 1.8 to 2.5 ). In a study of nearly 2,000 high school students in Southern California, almost 10\% of whom had used electronic cigarettes in the last 30 days, it was associated with an almost twofold increase in the risk of chronic bronchial symptoms (chronic cough, sputum production or bronchitis) [12]. 
Reporting of respiratory symptoms by e-cigarette users suggests an increased susceptibility to respiratory infections.

The factors that increase the risk of inflammatory processes, including infection, consist of inter alia, impaired cleansing of the mucociliary apparatus or impaired phagocytosis of neutrophils and macrophages.

Analysis of the sputum of e-cigarette users shows a higher level of activation of neutrophils, including myeloperoxidase, neutrophilic elastase and proteinase-3, which is analogous to the effect of traditional cigarettes. In the case of dysregulation of protease functions in the lungs, the basement membranes may be destroyed, which, in the case of chronic exposure, may lead to emphysema [12].

It is estimated that smoking is the cause of approximately $90 \%$ of lung cancer incidence [13]. Secondhand smoke also increases the likelihood of developing lung cancer, which is the most common cancer in the world. Approximately 1.6 million people die from lung cancer each year [14].

The influence of nicotine and other compounds on the cardiovascular system

The mechanism of action of nicotine is based on binding to nicotinic cholinergic receptors, which are located in the brain, the autonomic ganglia and the adrenal medulla. Binding of nicotine to acetylcholine receptors causes the release of catecholamines, both locally and systemically. Nicotine also acts on non-neuronal acetylcholine receptors, which are found on endothelial cells, inflammatory cells, macrophages and keratinocytes. Long-term exposure to nicotine causes loss of receptor sensitivity and leads to the development of high tolerance to its high concentration. Long-term exposure to nicotine causes loss of receptor sensitivity, increased tolerance to its high concentration, which in turn leads to the development of addiction [15].

The potential harmful effects of nicotine on the cardiovascular system can be assessed by assessing the changes in smokers who have decided to try to quit smoking with nicotine replacement therapy. According to the analyzed studies, nicotine replacement therapy in patients with cardiovascular disease does not increase the risk of cardiovascular events compared to placebo. Moreover, a meta-analysis of 21 clinical trials confirmed that nicotine replacement therapy is not associated with an increased risk of serious adverse cardiac reactions compared to placebo, but is associated with an increased risk of less serious reactions, such as palpitations [15].

Glantz and Bareham [16] describe the influence of e-cigarettes on the respiratory and vascular systems as just as harmful as ordinary cigarettes. E-cigarettes work by creating an aerosol of fine particles that transports nicotine to the lungs. The smallest biologically active particles trigger inflammatory processes, activate platelets, their aggregation and adhesion, which may induce acute cardiovascular reactions [17]. Additionally, e-cigarettes expose users to acrolein and other aldehydes, which irritate the mucous membranes and upper respiratory tract and, worse, contribute to the development of lung cancer. 
As with cardiovascular disease, evidence has consistently shown that exposure to e-cigarette aerosol has adverse effects on lungs and lung function. Repeated exposure to acrolein, which is produced by heating propylene glycol and glycerin in e-liquids, causes chronic pneumonia, progressive impairment of the immune system, excessive mucus secretion, and proteasemediated lung tissue damage that has been linked to the development of chronic obstructive pulmonary disease.

The effect of traditional cigarettes on the cardiovascular system.

In addition to nicotine, cigarette smoke includes products of tobacco combustion such as polycyclic aromatic hydrocarbons (e.g. benzopyrene), heavy metals (e.g. lead, cadmium, chromium, copper) and carbon monoxide. The chemicals in tobacco smoke are the major contributors to atherogenesis and thrombosis. The cardiovascular effects of cigarette smoking include the progression and worsening of heart failure, hypertensive heart disease, arrhythmic events including atrial fibrillation and sudden cardiac death. Echocardiography shows an increased incidence of left ventricular hypertrophy and diastolic dysfunction. In addition, tobacco smoke oxidants damage endothelial cells, reduce nitric oxide bioavailability, deplete endogenous antioxidants, cause inflammation, and generate highly atherogenic low-density lipoprotein (LDL) oxidized particles. Cigarette smoke can also contain large amounts of carbon monoxide [15].

George et al. [18] conducted a prospective, controlled VESUVIUS study involving 114 people who had to meet certain criteria: >= 18 years of age, smoking a minimum of 15 cigarettes a day for at least 2 years, and no history of cardiovascular disease. The methodology of the test consisted in replacing traditional cigarettes with their electronic substitutes, and then the evaluation and comparative analysis of the results of vessel functionality in the group of subjects after a month. The methods that were used to assess the vascular system were the measurement of flow-dependent dilation (FMD) and pulse wave velocity [4].

A meta-analysis of the FMD measurement results showed a 13\% lower adjusted relative risk of cardiovascular events for each 1\% improvement in FMD. Cigarette smokers who switched to electronic cigarettes improved their vascular functionality, approaching the values observed in healthy non-smokers (1.5\% improvement within a month) [18].

Research on the exposure of e-cigarette users suggests that e-cigarette use can lead to oxidative stress, the role of which in the pathophysiology of cardiovascular disease is well understood. As a result, the quantification of reactive oxygen species as a promising biomarker reflecting the disease process is being sought.

In a study by Carnevale et al. [19] there were 40 participants with a negative medical history (no fever and infection in the last 3 months, no history of cardiovascular diseases, no allergies, normal blood pressure, normal heart rhythm). The study consisted of smoking traditional cigarettes by the entire group of respondents (the first week of the trial), and then smoking their electronic substitutes (the second week of the trial). 
The amount of nicotine was approximately the same when smoking a conventional cigarette $(0.6 \mathrm{mg})$ and an e-cigarette $(0.6 \mathrm{mg}$ with 9 puffs). Blood sampling and FMD measurement took place just before and 30 minutes after smoking. In this study, the influence of traditional cigarettes and e-cigarettes on the development of oxidative stress and vascular endothelial cell dysfunction was assessed and analyzed. Both after smoking classic and electronic cigarettes, there was a decrease in the level of vitamin E (antioxidant), a decrease in the bioavailability of nitric oxide (NO), as well as a significant increase in the biomarkers of oxidative stress: NADPH oxidase (sNox2-dp), prostaglandin 2 alpha (8-isoPGF2alpha). Statistical significance for all of them was $P<0.001$. However, the interaction analysis showed that e-cigarettes had a much smaller effect than conventional cigarettes on the levels of NADPH oxidase $(\mathrm{P}=0.001)$, prostaglandin 2 alpha $(\mathrm{P}=0.046)$ and NO bioavailability $(\mathrm{P}=0.001)$ [19].

Other exposures arising from the use of e-cigarettes

Changing behavior or misuse of e-cigarettes may result in increased nicotine exposure. De Vito and Krishnan-Sarin [20] suggest that nicotine re-exposure from e-cigarettes is possible. It has been shown that there is a possibility of exposure to nicotine from e-cigarettes, which can be absorbed through the skin. Importantly, the nicotine levels of e-cigarette aerosol can be similar to or significantly higher than that found in conventional cigarettes.

The next questions were posed by Giovenco and Delnevo [21] - Can e-cigarettes help you quit smoking? The results of the study showed that frequent or long-term use of e-cigarettes can contribute to smoking cessation. The authors do not forget about the so-called "double smokers", that is, those who alternate between cigarettes and e-cigarettes, thereby increasing the exposure to inhalation of various harmful substances in greater amounts. Overall, the link between e-cigarettes and quitting smoking is inconclusive, but the findings suggest that using them may play a role in quitting, which would affect the positive aspect of choosing ecigarettes over regular cigarettes.

As is clear from the works of various authors presented above, e-cigarettes are harmful and, additionally, they can be used to smoke substances other than nicotine or in combination with it.

The use of nicotine-containing solutions and THC-enriched e-liquids (Tetrahydrocannabinol THC) may promote dual addiction, as in the above-mentioned article. The side effects of chronic use of these substances are more dangerous than regular cigarettes. They can cause a lower gray matter volume in the brain or a cognitive decline. It's also worth noting that after Butane Honey Oil $(\mathrm{BHO})$ has evaporated, it remains largely unexplored in the e-liquid. There is a belief in society that the use of e-cigarettes with $\mathrm{BHO}$ is more harmful than the use of other forms of cannabis [22].

A newly registered FDA (April 2019) product is a non-burning IQOS product that works by heating tobacco rather than smoking it. According to its own research, PMI (Philip Morris International Inc. - an American company with a 15.5\% share in the global tobacco market outside the US) found that IQOS contains much less (about 90\%) harmful and potentially harmful ingredients (HPHC) compared to cigarettes [23]. 
It is worrying that interest in these devices is growing among adolescents, and nicotine exposure is higher among e-cigarette users than among regular cigarette smokers [24].

\section{Summary}

An undeniable problem is that the positive marketing surrounding e-cigarettes and other alternative forms of tobacco smoking is encouraging younger and younger users to reach for them [24]. The temporary fashion for e-cigarettes often ends with the desire to try a classic cigarette, being the first step to a long-term addiction. Gradually giving up cigarettes at the expense of electronic alternatives may be one way to quit smoking altogether. However, there are cases of people who, while trying to do so, started smoking both traditional cigarettes and their electronic substitutes.

Electronic cigarettes are still new to the tobacco product market. They were supposed to be an option for smokers who, despite their addiction, care about their health and try to gradually give up smoking. And also encourage many non-smokers to try the e-cigarette. But is the ecigarette really a better alternative, "the lesser evil" compared to traditional cigarettes? Public Health England, the Royal College of Physicians, the Royal Society for Public Health, and the National Health Service [footnote-26] have stated clearly that e-cigarettes are 95\% safer than conventional cigarettes. It seems, however, that it is too early for unequivocal opinions. We need more years of research on large groups of patients that will help us answer this question.

Contribution of authors:

M. Romaniuk - study concept and design; critical revision of the manuscript for important intellectual content; study supervision;

K. Suswat - acquisition of data; analysis and interpretation of data; technical support;

M. Komisarczuk - acquisition of data; analysis and interpretation of data; technical support;

W. Tuszyńska - acquisition of data; analysis and interpretation of data; technical support

K. Krupa- acquisition of data; analysis and interpretation of data; technical support

W. Zygmunt - acquisition of data; analysis and interpretation of data; technical support

J. Milanowski - critical revision of the manuscript for important intellectual content; study supervision.

\section{Disclosures}

Financial support: No financial support was received.

Conflict of interest: The authors declare no financial or other conflict of interest. 


\section{Bibliography:}

1. Gately I. Tobacco: A Cultural History of How an Exotic Plant Seduced Civilization, Diane, 2004; 3-7.

2. Dela Cruz CS., Tanoue LT., Matthay RA. Lung cancer: epidemiology, etiology, and prevention. Clin Chest Med. 2011; 32(4): 605-644.

3. 1.World Health Organization. Tobacco. https://www.who.int/news-room/factsheets/detail/tobacco [Available: 2020 may 27]

4. Bialous SA., Sarna L. Lung Cancer and Tobacco: What Is New?. Nurs Clin North Am. 2017;52(1):53-63. doi:10.1016/j.cnur.2016.10.003

5. World Health Organization. Cancer Prevention. https://www.who.int/cancer/prevention/en/

6. Lik H. Electronic Atomization Cigarette. United States: [Available: 26 Apr 2016]. Google Patents, assignee. http://www.google.com/patents/US20070267031.

7. Williams M, Talbot P. Variability among electronic cigarettes in the pressure drop, airflow rate, and aerosol production. Nicotine Tob Res. 2011;13(12):1276-1283.

8. Hess CA, Olmedo P, Navas-Acien A, Goessler W, Cohen JE, Rule AM. E-cigarettes as a source of toxic and potentially carcinogenic metals. Environ Res. 2017;152:221-225. doi:10.1016/j.envres.2016.09.026

9. Allen JG, Flanigan SS, LeBlanc M, et al. Flavoring Chemicals in E-Cigarettes: Diacetyl, 2,3-Pentanedione, and Acetoin in a Sample of 51 Products, Including Fruit-, Candy-, and Cocktail-Flavored E-Cigarettes. Environ Health Perspect. 2016;124(6):733-739. doi:10.1289/ehp.1510185

10. Clapp PW, Jaspers I. Electronic cigarettes: Their constituents and potential links to asthma. CurrAllergy Asthma Rep 2017;17(11):79.

11. Reinikovaite V, Rodriguez IE, Karoor V, et al. The effects of electronic cigarette vapour on the lung: direct comparison to tobacco smoke. Eur Respir J 2018; 4;51(4):1701661. doi: 10.1183/13993003.01661-2017.

12. Gotts JE, Jordt SE, McConnell R, et al. What are the respiratory effects of ecigarettes? BMJ 2019; 366:15275. doi: 10.1136/bmj.15275

13. Moyer V.A. Screening for Lung Cancer: U.S. Preventive Services Task Force Recommendation Statement. Ann Intern Med. 2014;160(5):330-338. ]

14. Stewart BW. and Wild CP. World Cancer Report 2014. World Health Organization, 2014.

15. Benowitz NL, Fraiman JB. Cardiovascular effects of electronic cigarettes. Nat Rev Cardiol. 2017;14(8):447-456. doi: 10.1038/nrcardio.2017.36.

16. Glantz SA, Bareham DW. E-Cigarettes: Use, Effects on Smoking, Risks, and Policy Implications. Annu Rev Public Health. 2018;39:215-235. doi:10.1146/annurev-publhealth040617-013757

17. Schweitzer RJ, Wills TA, Behner D. E-cigarette use and indicators of cardiovascular disease risk. Curr. Epid. Rep 2017;4:248-57.

18. George J, Hussain M, Vadiveloo T, et al. Cardiovascular Effects of Switching From Tobacco Cigarettes to Electronic Cigarettes. J Am Coll Cardiol. 2019;74(25):3112-3120. doi:10.1016/j.jacc.2019.09.067 
19. Carnevale R, Sciarretta S, Violi F, et al. Acute Impact of Tobacco vs Electronic Cigarette Smoking on Oxidative Stress and Vascular Function. Chest. 2016;150(3):606-612. doi:10.1016/j.chest.2016.04.012

20. DeVito EE., Krishnan-Sarin S. E-cigarettes: Impact of E-liquid Components and Device Characteristics on Nicotine Exposure. Current Neuropharmacology, 2018; 16, 438459.

21. Giovenco DP., Delnevo CD., Prevalence of population smoking cessation by electronic cigarette use status in a national sample of recent smokers. Columbia University Mailman School of Public Health, Department of Sociomedical Sciences, 2018;76: 129-134. doi:10.1016/j.addbeh.2017.08.002.

22. Giroud C., Cesare M., Berthet A.,Varlet V., Concha-Lozano N., Favrat B., ECigarettes: A Review of New Trends in Cannabis Use. Int. J. Environ. Res. Public Health 2015;12, 9988-10008; doi:10.3390/ijerph120809988

23. Adriaens K., Gucht D., Baeyens F. IQOS vs. e-Cigarette vs. Tobacco Cigarette: A Direct Comparison of Short-Term Effects after Overnight-Abstinence. 2018;15(12):2902. doi: 10.3390/ijerph15122902

24. Boykan R, Goniewicz ML, Messina CR. Evidence of Nicotine Dependence in Adolescents Who Use Juul and Similar Pod Devices. Int J Environ Res Public Health. 2019;16(12):2135. Published 2019 Jun 17. doi:10.3390/ijerph16122135 